\title{
Decision of Lead-Time Compression and Stable Operation of Supply Chain
}

\author{
Songtao Zhang, ${ }^{1}$ Shuangshuang Li, ${ }^{2}$ Siqi Zhang, ${ }^{3}$ and Min Zhang ${ }^{4}$ \\ ${ }^{1}$ School of Logistics, Linyi University, Linyi 276005, China \\ ${ }^{2}$ School of Management, Harbin University of Commerce, Harbin 150028, China \\ ${ }^{3}$ Faculty of Business and Economics, The University of Melbourne, Melbourne, VIC 3010, Australia \\ ${ }^{4}$ Library, Linyi University, Linyi 276005, China \\ Correspondence should be addressed to Songtao Zhang; zst0626@163.com
}

Received 17 August 2017; Accepted 22 October 2017; Published 13 November 2017

Academic Editor: Danilo Comminiello

Copyright (c) 2017 Songtao Zhang et al. This is an open access article distributed under the Creative Commons Attribution License, which permits unrestricted use, distribution, and reproduction in any medium, provided the original work is properly cited.

\begin{abstract}
A cost optimization strategy and a robust control strategy were studied to realize the low-cost robust operation of the supply chain with lead times. Firstly, for the multiple production lead times which existed in the supply chain, a corresponding inventory state model and a supply chain cost model were constructed based on the Takagi-Sugeno fuzzy control system. Then, by considering the actual inventory level, the lead-time compression cost, and the stock-out cost, a cost optimization strategy was proposed. Furthermore, a fuzzy robust control strategy was proposed to realize the flexible switching among the models. Finally, the simulation results show that the total cost of the supply chain could be reduced effectively by the cost optimization strategy, and the stable operation of the supply chain could be realized by the proposed fuzzy robust control strategy.
\end{abstract}

\section{Introduction}

With the application of information technology and the intensification of global competition, price plays an increasingly important role in market competition [1, 2]. In addition, due to the acceleration of product updates, how to quickly respond to customers' demands in a short time has become a major factor that needs to be considered when enterprises provide products and services $[3,4]$. Therefore, the research on time management and cost management of supply chain has been widely concerned by many scholars [5-11]. Many scholars have studied from the perspective of lead time, that is, by compressing the lead time to achieve the purpose of cost reduction. Under the assumption that the lead time was composed of production time, setup time, and transportation time, Glock [9] confirmed that a mixture of setup time and production time reduction can effectively reduce the expected total costs. For the exponential lead times, Hayya et al. [11] demonstrated that the lead times reduction can lead to a reduction in the inventory cost.
The research results of the above literatures have proven that the cost of the supply chain can be reduced to a certain extent by compressing the lead time. However, when the inventory level is less than 0 , none of literatures have considered using the lower stock-out cost to replace the higher lead-time compression cost. Therefore, it is necessary to determine whether to compress the lead time according to the actual situation. In addition, the above literatures did not take into account the robustness of the supply chain system after compressing the lead time. In order to improve the robustness of the system, some scholars have applied the robust control method and the robust optimization method [12-19]. For a class of discrete systems with multiple delays and disturbances, Teng et al. [12] proposed a Takagi-Sugeno fuzzy approach to achieve the robustness of model predictive control. Compared with most of the existing methods for 3D path following, the robust fuzzy control scheme proposed by Xiang et al. [14] can be more effective in reducing the implementation costs of complicated dynamics controller and environmental disturbances. By taking account of the 
location effect, dispersion effect, and model uncertainty of the multiple responses simultaneously, He et al. [19] developed a robust fuzzy programming approach to solve the multiple responses optimization problems, which can ensure the robustness of the system.

Although the above-mentioned literatures have improved the robustness of the supply chain system after the application of the robust method, the literatures did not take into account the fact that the node enterprises of the supply chain will take the corresponding production strategy and ordering strategy to reduce the total cost of the supply chain system under the different inventory levels.

Therefore, in this paper, by combining with the production and ordering strategies of the node enterprises under different inventory levels, we will first construct a total cost model and an inventory model of the supply chain considering the lead-time compression. What is more, in order to minimize the total operation cost of the supply chain, a cost optimization strategy will be proposed by comparing the lead-time compression cost and the stock-out cost at different inventory levels. Finally, a fuzzy robust control strategy is proposed to suppress the disturbances and achieve the low-cost stable operation of the supply chain system.

This paper is structured as follows: Section 2 constructs a discrete fuzzy model of the dynamic supply chain system with lead times and proposes a cost optimization strategy. In Section 3, a fuzzy robust control strategy for the dynamic supply chain with lead times is proposed. Section 4 gives a simulation example to illustrate the effectiveness of the proposed strategies. Section 5 presents some conclusions.

\section{Model Description}

2.1. Model of the Compression Cost for Lead Time. In order to analyze the quantitative relation between the compression cost and the compression amount for the lead time, we construct a model of the compression cost for the lead time as follows:

$$
c_{\tau}=c_{i}\left(\tau_{i-1}-\tau\right)+\sum_{j=1}^{i-1} c_{j}\left(b_{j}-a_{j}\right), \quad \tau \in\left[\tau_{i}, \tau_{i-1}\right] .
$$

Model (1) adopts the segmental cumulative calculation method, which starts from the part with the minimum compression cost, calculates the compression cost of each independent part of lead times in turn, and adds up the compression cost of each independent part. In Model (1), $c_{\tau}$ is the total compression cost of the lead time; $\tau$ is the lead time existing in each operating sector of the supply chain; $\tau_{i}$ is the length of the lead time after compressing one to $i$ components, $i=1,2, \ldots, n$; $c_{i}$ is the unit compression cost of the $i$ th component of the lead time; $b_{i}$ is the standard operation time of the $i$ th component of the lead time; $a_{i}$ is the shortest operation time of the $i$ th component of the lead time after complete compression.

2.2. Model of Dynamic Supply Chain System with Lead Times. We construct a dynamic supply chain system including $n-1$ manufacturers, a retailer, and the customers. The detailed constructions of the supply chain system can be seen in Figure 1.

In Figure $1, x_{a}(k)$ and $x_{n}(k)$ are the manufacturer $a$ 's inventory and the retailer's inventory at period $k$, respectively; $x_{a}(k)$ and $x_{n}(k)$ are the state variables; $w_{1}(k)$ is the customers' demands at period $k ; w_{1}(k)$ is the external disturbance variable; $u_{a n}(k)$ is the ordering quantity from the retailer to manufacturer $a$ at period $k ; u_{a n}(k)$ is the control variable; $u_{a}^{\prime}(k)$ is the manufacturer $a$ 's production at period $k$ under different production strategies, which can be expressed as follows: (1) $u_{a}^{\prime}(k)=u_{a}(k)+u_{a}\left(k-\tau_{a}^{\prime}\right)$, where $u_{a}(k)$ is the manufacturer $a^{\prime}$ s production at period $k, u_{a}\left(k-\tau_{a}^{\prime}\right)$ is the manufacturer $a$ 's production within the lead time $\tau_{a}^{\prime}$, and $\tau_{a}^{\prime}$ is the initial production lead time; $(2) u_{a}^{\prime}(k)=u_{a}\left(k-\tau_{a}^{\prime \prime}\right)$, where $u_{a}\left(k-\tau_{a}^{\prime \prime}\right)$ is the manufacturer $a$ 's production within the lead time $\tau_{a}^{\prime \prime} ; \tau_{a}^{\prime \prime}$ is the compressed production lead time. We will select one of the different expressions about $u_{a}^{\prime}(k)$ according to different circumstances, and the specific selection criteria can be seen in Note 1 .

According to the system structure in Figure 1, the inventory state model and the total cost model of the supply chain system with lead times can be constructed as follows:

$$
\begin{aligned}
& x_{a}(k+1)=x_{a}(k)+u_{a}(k)+u_{a}\left(k-\tau_{a}^{\prime}\right)+u_{a}(k \\
& \left.-\tau_{a}^{\prime \prime}\right)-u_{a n}(k), \quad a=1,2, \ldots, n-1, \\
& x_{n}(k+1)=x_{n}(k)+\sum_{a=1}^{n-1} u_{a n}(k)-w_{1}(k), \\
& z(k)=\sum_{a=1}^{n-1}\left\{c_{h a} x_{a}(k)+c_{n a}\left[u_{a}(k)+u_{a}\left(k-\tau_{a}^{\prime}\right)\right]\right. \\
& \left.+c_{\tau a} u_{a}\left(k-\tau_{a}^{\prime \prime}\right)+c_{s a} u_{a n}(k)+c_{m a} u_{a n}(k)\right\} \\
& +c_{h n} x_{n}(k),
\end{aligned}
$$

where $z(k)$ is the total operating cost of the dynamic supply chain system at period $k$; $c_{h a}$ is the manufacturer $a$ 's unit inventory cost; $c_{n a}$ is the manufacturer $a$ 's unit production cost; $c_{\tau a}$ is the manufacturer $a$ 's unit compression cost by selecting the compression strategy; $c_{s a}$ is the unit ordering cost from the retailer to manufacturer $a$; $c_{m a}$ is the manufacturer $a$ 's unit stock-out cost by selecting the out of stock strategy; $c_{h n}$ is the retailer's unit inventory cost.

Note 1. The cost optimization strategy proposed in this paper includes the following two aspects: (1) The inventory level is less than the safety inventory: if the lead-time compression cost is less than the stock-out cost, then the lead time is compressed, the variables $u_{a}(k)$ and $u_{a}\left(k-\tau_{a}^{\prime}\right)$ do not exist, and the coefficients $c_{n a}$ and $c_{m a}$ are 0 ; if not, the lead time is not compressed, the variable $u_{a}\left(k-\tau_{a}^{\prime \prime}\right)$ does not exist, and the coefficients $c_{\tau a}$ and $c_{s a}$ are 0 ; (2) the inventory level is greater than the safety inventory: the node enterprises can produce and order the products normally, the variable $u_{a}\left(k-\tau_{a}^{\prime \prime}\right)$ does not exist, and the coefficients $c_{\tau a}$ and $c_{m a}$ are 0 . 


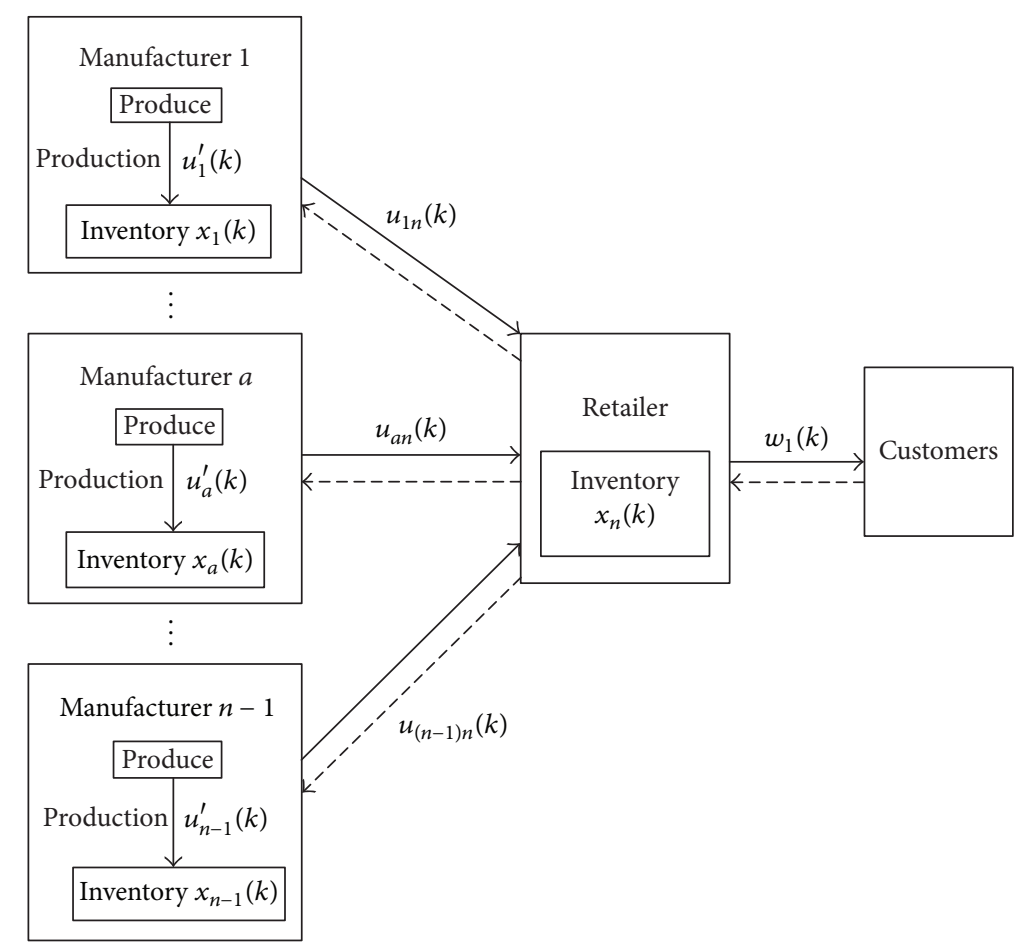

FIGURE 1: Dynamic supply chain system with lead times.

In order to reduce the total cost of the supply chain system, the manufacturer and the retailer will adopt the corresponding production strategy and ordering strategy under different inventory levels. Therefore, different submodels will be formed in the established supply chain system. In the following, the ith submodel is obtained by matrix transformation of Model (2):

$$
\begin{aligned}
\mathbf{x}(k+1)= & \mathbf{A}_{i} \mathbf{x}(k)+\mathbf{B}_{i} \mathbf{u}(k)+\sum_{a=1}^{2(n-1)} \mathbf{B}_{i a} \mathbf{u}\left(k-\tau_{a}\right) \\
& +\mathbf{B}_{w i} \mathbf{w}(k), \\
z(k)= & \mathbf{C}_{i} \mathbf{x}(k)+\mathbf{D}_{i} \mathbf{u}(k)+\sum_{a=1}^{2(n-1)} \mathbf{D}_{i a} \mathbf{u}\left(k-\tau_{a}\right),
\end{aligned}
$$

where $\mathbf{x}(k)=\left[x_{1}(k), \ldots, x_{a}(k), \ldots, x_{n}(k)\right]^{\mathrm{T}}$ is the inventory state vector at period $k ; \mathbf{u}(k)=\left[u_{1}(k), \ldots, u_{a}(k), \ldots, u_{n-1}(k)\right.$, $\left.u_{1 n}(k), \ldots, u_{a n}(k), \ldots, u_{(n-1) n}(k)\right]^{\mathrm{T}}$ is the production and ordering control vector at period $k ; \mathbf{u}^{\mathrm{T}}\left(k-\tau_{a}\right)=\left[\mathbf{u}^{\mathrm{T}}(k-\right.$ $\left.\left.\tau_{a}^{\prime}\right), \mathbf{u}^{\mathrm{T}}\left(k-\tau_{a}^{\prime \prime}\right)\right], \mathbf{u}\left(k-\tau_{a}^{\prime}\right)=\left[u_{1}\left(k-\tau_{1}^{\prime}\right), \ldots, u_{a}(k-\right.$ $\left.\left.\tau_{a}^{\prime}\right), \ldots, u_{(n-1)}\left(k-\tau_{n-1}^{\prime}\right)\right]^{\mathrm{T}}$ is the production control vector within the initial production lead time $\tau_{a}^{\prime}, \mathbf{u}\left(k-\tau_{a}^{\prime \prime}\right)=\left[u_{1}(k-\right.$ $\left.\left.\tau_{1}^{\prime \prime}\right), \ldots, u_{a}\left(k-\tau_{a}^{\prime \prime}\right), \ldots, u_{n-1}\left(k-\tau_{n-1}^{\prime \prime}\right)\right]^{\mathrm{T}}$ is the production control vector within the compressed production lead time $\tau_{a}^{\prime \prime} ; \mathbf{w}(k)=\left[0,0, \ldots, w_{1}(k)\right]^{\mathrm{T}}$ is the customers' demands vector at period $k ; \mathbf{A}_{i}$ is the inventory coefficient matrix; $\mathbf{B}_{i}$ is the production and ordering coefficient matrix; $\mathbf{B}_{i a}$ is the production coefficient matrix with lead time, $\sum_{a=1}^{n-1} \mathbf{B}_{i a}$ is the production coefficient matrix of $n-1$ manufacturers within the initial production lead time $\tau_{a}^{\prime}$, and $\sum_{a=n}^{2(n-1)} \mathbf{B}_{i a}$ is the production coefficient matrix of $n-1$ manufacturers within the compressed production lead time $\tau_{a}^{\prime \prime} ; \mathbf{B}_{w i}$ is the uncertain customers' demands coefficient matrix of the supply chain system; $\mathbf{C}_{i}$ is the inventory cost coefficient matrix; $\mathbf{D}_{i}$ is the production and ordering cost coefficient matrix (or the stock-out cost coefficient matrix); $\mathbf{D}_{i a}$ is the production cost coefficient matrix with lead time; $\sum_{a=1}^{n-1} \mathbf{D}_{i a}$ is the production cost coefficient matrix of $n-1$ manufacturers within the initial production lead time $\tau_{a}^{\prime} ; \sum_{a=n}^{2(n-1)} \mathbf{D}_{i a}$ is the production cost coefficient matrix of $n-1$ manufacturers within the compressed production lead time $\tau_{a}^{\prime \prime}$.

2.3. Takagi-Sugeno Fuzzy Model of Dynamic Supply Chain System with Lead Times. As mentioned above, the dynamic supply chain system with lead times constructed in this paper contains different submodels. As the inventory level changes, the switching actions will occur among the submodels, which will lead to the fluctuations of the variables in the supply chain system. In order to effectively suppress the fluctuations of each variable and achieve the flexible switching among the submodels, by utilizing Takagi-Sugeno fuzzy control system, the fuzzy control model of dynamic supply chain system with lead times can be constructed by the following fuzzy IFTHEN rules: then

$$
R_{i} \text { : if } x_{1}(k) \text { is } M_{1}^{i}, \ldots, x_{j}(k) \text { is } M_{j}^{i}, \ldots \text {, and } x_{n-1}(k) \text { is } M_{n-1}^{i} \text {, }
$$

$$
\begin{aligned}
\mathbf{x}(k+1)= & \mathbf{A}_{i} \mathbf{x}(k)+\mathbf{B}_{i} \mathbf{u}(k) \\
& +\sum_{a=1}^{2(n-1)} \mathbf{B}_{i a} \mathbf{u}\left(k-\tau_{a}\right)+\mathbf{B}_{w i} \mathbf{w}(k),
\end{aligned}
$$




$$
\begin{aligned}
& z(k)=\mathbf{C}_{i} \mathbf{x}(k)+\mathbf{D}_{i} \mathbf{u}(k)+\sum_{a=1}^{2(n-1)} \mathbf{D}_{i a} \mathbf{u}\left(k-\tau_{a}\right), \\
& \mathbf{x}(k)=\boldsymbol{\varphi}(k),
\end{aligned}
$$

$$
i=1,2, \ldots, r, k \in\{0,1, \ldots, N\} \text {, }
$$

where $R_{i}(i=1,2, \ldots, r)$ is the $i$ th control rule of the fuzzy system and $r$ is the number of IF-THEN rules; $M_{j}^{i}(j=$ $1,2, \ldots, n-1)$ is the fuzzy set; $\varphi(k)$ is the initial condition of the dynamic supply chain with lead times.

Based on singleton fuzzification, product inference, and center-average defuzzification, Model (4) can be inferred as follows:

$$
\begin{aligned}
& \mathbf{x}(k+1)=\sum_{i=1}^{r} h_{i}(\mathbf{x}(k))\left[\mathbf{A}_{i} \mathbf{x}(k)+\mathbf{B}_{i} \mathbf{u}(k)\right. \\
& \left.+\sum_{a=1}^{2(n-1)} \mathbf{B}_{i a} \mathbf{u}\left(k-\tau_{a}\right)+\mathbf{B}_{w i} \mathbf{w}(k)\right], \\
& z(k)=\sum_{i=1}^{r} h_{i}(\mathbf{x}(k))\left[\mathbf{C}_{i} \mathbf{x}(k)+\mathbf{D}_{i} \mathbf{u}(k)\right. \\
& \left.+\sum_{a=1}^{2(n-1)} \mathbf{D}_{i a} \mathbf{u}\left(k-\tau_{a}\right)\right],
\end{aligned}
$$

where $h_{i}(\mathbf{x}(k))=\mu_{i}(\mathbf{x}(k)) / \sum_{i=1}^{r} \mu_{i}(\mathbf{x}(k)), \mu_{i}(\mathbf{x}(k))=$ $\prod_{j=1}^{n-1} M_{j}^{i}\left(x_{j}(k)\right), M_{j}^{i}\left(x_{j}(k)\right)$ is the grade of membership of $x_{j}(k)$ in the fuzzy set $M_{j}^{i}, \mu_{i}(\mathbf{x}(k))$ is the membership degree of the $i$ th rule, and $h_{i}(\mathbf{x}(k))$ is abbreviated to $h_{i}$ in the following.

\section{Fuzzy Robust Control of Dynamic Supply Chain System}

For the impacts of the lead time and the uncertain external demands on the operation of the supply chain system, the fuzzy robust control approach can guarantee the robust stable operation of the dynamic supply chain system by controlling the production variables and ordering variables through the inventory state variables. In this paper, the parameter $\gamma$ is introduced to represent the suppression degree of the supply chain system for the above-mentioned disturbances, which can be expressed as follows:

$$
\frac{\left\|\sum_{i=1}^{r} h_{i}(\mathbf{x}(k))\left[\mathbf{C}_{i} \mathbf{x}(k)+\mathbf{D}_{i} \mathbf{u}(k)+\sum_{a=1}^{2(n-1)} \mathbf{D}_{i a} \mathbf{u}\left(k-\tau_{a}\right)\right]\right\|_{2}}{\|\mathbf{w}(k)\|_{2}}
$$

$$
\leq \gamma
$$

where $\|\cdot\|_{2}$ is $l_{2} \in[0, \infty)$. Inequality (6) describes the system gain characteristic from the customers' demands to the total cost of the dynamic supply chain. The smaller the parameter $\gamma$ is, the stronger the ability of the system to suppress the disturbances will be.

The local feedback controller for each submodel of the dynamic supply chain fuzzy system can be designed as follows.

Controller Rule $K^{i}$ : if $x_{1}(k)$ is $M_{1}^{i}, \ldots, x_{j}(k)$ is $M_{j}^{i}, \ldots$, and $x_{n-1}(k)$ is $M_{n-1}^{i}$, then

$$
\begin{gathered}
\mathbf{u}(k)=-\mathbf{K}_{i} \mathbf{x}(k), \\
\mathbf{u}\left(k-\tau_{a}\right)=-\mathbf{K}_{i a} \mathbf{x}\left(k-\tau_{a}\right),
\end{gathered}
$$

where $\mathbf{K}_{i}$ is the state feedback constant gain matrix and $\mathbf{K}_{i a}$ is the state feedback constant gain matrix with lead times. Then, the overall state feedback controller of the supply chain system can be expressed as follows:

$$
\begin{gathered}
\mathbf{u}(k)=-\sum_{i=1}^{r} h_{i} \mathbf{K}_{i} \mathbf{x}(k), \\
\mathbf{u}\left(k-\tau_{a}\right)=-\sum_{i=1}^{r} h_{i} \mathbf{K}_{i a} \mathbf{x}\left(k-\tau_{a}\right) .
\end{gathered}
$$

Thus, (5) can be further expressed as

$$
\begin{gathered}
\mathbf{x}(k+1)=\sum_{i=1}^{r} \sum_{j=1}^{r} h_{i} h_{j}\left[\left(\mathbf{A}_{i}-\mathbf{B}_{i} \mathbf{K}_{j}\right) \mathbf{x}(k)\right. \\
\left.-\sum_{a=1}^{2(n-1)} \mathbf{B}_{i a} \mathbf{K}_{j a} \mathbf{x}\left(k-\tau_{a}\right)+\mathbf{B}_{w i} \mathbf{w}(k)\right], \\
z(k)=\sum_{i=1}^{r} \sum_{j=1}^{r} h_{i} h_{j}\left[\left(\mathbf{C}_{i}-\mathbf{D}_{i} \mathbf{K}_{j}\right) \mathbf{x}(k)\right. \\
\left.-\sum_{a=1}^{2(n-1)} \mathbf{D}_{i a} \mathbf{K}_{j a} \mathbf{x}\left(k-\tau_{a}\right)\right] .
\end{gathered}
$$

Two Definitions are introduced as follows.

Definition 1 (see [20]). A cluster of fuzzy sets $\left\{F_{j}^{m}, m=\right.$ $\left.1,2, \ldots, q_{j}\right\}$ are said to be a standard fuzzy partition (SFP) in the universe $X$ if each $F_{j}^{m}\left(m=1,2, \ldots, q_{j}\right)$ is full-overlapped in the universe $X . q_{j}$ is said to be the number of fuzzy partitions of the $j$ th input variable on $X$.

Definition 2 (see [20]). For a given fuzzy system, an overlapped-rules group with the largest amount of rules is said to be a maximal overlapped-rules group (MORG).

In order to realize the robustly stable operation of the supply chain fuzzy system (9), the modified Theorem 2 proposed by us in [21] can be seen as follows.

Theorem 3. For the supply chain fuzzy system (9) with lead times and SFP inputs, if there exists a given scalar $\gamma>$ 0 , local common positive definite matrices $\mathbf{P}_{c}$ and $\mathbf{Q}_{a c}$, and matrices $\mathbf{K}_{i c}, \mathbf{K}_{j c}, \mathbf{K}_{i a c}, \mathbf{K}_{j a c}$ in $\mathbf{G}_{c}$, such that the following linear 


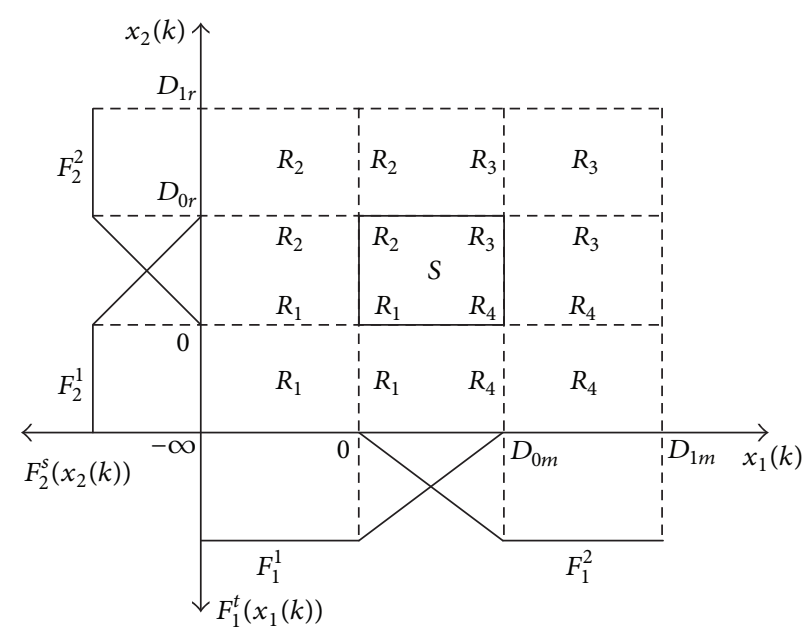

FIGURE 2: The sketch map of the fuzzy membership functions.

matrix inequalities (LMIs) are satisfied, then the supply chain fuzzy system (9) is robustly asymptotically stable under the performance $\gamma$ :

$$
\begin{aligned}
& {\left[\begin{array}{ccccc}
-\mathbf{P}_{c}+\sum_{a=1}^{2(n-1)} \mathbf{Q}_{a c} & * & * & * & * \\
\mathbf{0} & -\widehat{\mathbf{Q}} & * & * & * \\
\mathbf{0} & \mathbf{0} & -\gamma^{2} \mathbf{I} & * & * \\
\mathbf{A}_{i}-\mathbf{B}_{i} \mathbf{K}_{i c} & -\boldsymbol{\Pi}_{1} & \mathbf{B}_{w i} & -\mathbf{P}_{c} & * \\
\mathbf{C}_{i}-\mathbf{D}_{i} \mathbf{K}_{i c} & -\boldsymbol{\Pi}_{2} & \mathbf{0} & \mathbf{0} & -\mathbf{I}
\end{array}\right]<\mathbf{0}, \quad i \in I_{c},} \\
& {\left[\begin{array}{ccccc}
-4 \mathbf{P}_{c}+4 \sum_{a=1}^{2(n-1)} \mathbf{Q}_{a c} & * & * & * & * \\
\mathbf{0} & -4 \widehat{\mathbf{Q}} & * & * & * \\
\mathbf{0} & \mathbf{0} & -4 \gamma^{2} \mathbf{I} & * & * \\
\mathbf{A}_{i}-\mathbf{B}_{i} \mathbf{K}_{j c}+\mathbf{A}_{j}-\mathbf{B}_{j} \mathbf{K}_{i c} & -\boldsymbol{\Phi}_{1} & \mathbf{B}_{w i}+\mathbf{B}_{w j} & -\mathbf{P}_{c} & * \\
\mathbf{C}_{i}-\mathbf{D}_{i} \mathbf{K}_{j c}+\mathbf{C}_{j}-\mathbf{D}_{j} \mathbf{K}_{i c} & -\mathbf{\Phi}_{2} & \mathbf{0} & \mathbf{0} & -\mathbf{I}
\end{array}\right]}
\end{aligned}
$$

$<\mathbf{0}, \quad i<j, i, j \in I_{c}$,

where $\widehat{\mathbf{Q}}=\operatorname{diag}\left\{\begin{array}{lllll}\mathbf{Q}_{1 c} & \cdots & \mathbf{Q}_{a c} & \cdots & \mathbf{Q}_{2(n-1) c}\end{array}\right\}, \Pi_{1}=$ $\left[\begin{array}{llllll}\mathbf{B}_{i 1} \mathbf{K}_{i 1 c} & \cdots & \mathbf{B}_{i a} \mathbf{K}_{i a c} & \cdots & \mathbf{B}_{i 2(n-1)} \mathbf{K}_{i 2(n-1) c}\end{array}\right], \quad \boldsymbol{\Pi}_{2}=$ $\left[\begin{array}{llllll}\mathbf{D}_{i 1} \mathbf{K}_{i 1 c} & \cdots & \mathbf{D}_{i a} \mathbf{K}_{i a c} & \cdots & \mathbf{D}_{i 2(n-1)} \mathbf{K}_{i 2(n-1) c}\end{array}\right], \boldsymbol{\Phi}_{1}=\left[\mathbf{B}_{i 1} \mathbf{K}_{j 1 c}+\right.$ $\mathbf{B}_{j 1} \mathbf{K}_{i 1 c} \cdots \mathbf{B}_{i a} \mathbf{K}_{j a c}+\mathbf{B}_{j a} \mathbf{K}_{i a c} \cdots \mathbf{B}_{i 2(n-1)} \mathbf{K}_{j 2(n-1) c}+$

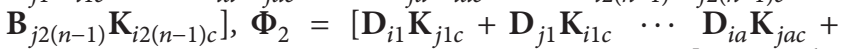
$\left.\mathbf{D}_{j a} \mathbf{K}_{i a c} \cdots \mathbf{D}_{i 2(n-1)} \mathbf{K}_{j 2(n-1) c}+\mathbf{D}_{j 2(n-1)} \mathbf{K}_{i 2(n-1) c}\right], I_{c}$ is the set of the rule numbers included in $\mathbf{G}_{c}, \mathbf{G}_{c}$ denotes the cth $M O R G, c=1,2, \ldots, \prod_{j=1}^{n}\left(m_{j}-1\right)$, and $m_{j}$ is the number of the fuzzy partitions of the jth input variable.

Proof. The proof processes of Theorem 3 are the same as those of Theorem 2 in [21].

\section{Simulation Analysis}

In this section, we will select a type of sports shoes supply chain system with two manufacturers and a retailer as the simulation object to analyze the effectiveness of the proposed cost optimization strategy and the fuzzy robust control strategy.

The semi-trapezoid membership functions are adopted as the fuzzy membership functions of the two sports shoes manufacturers in the simulation, which can be seen in Figure 2.

$x_{1}(k)$ and $x_{2}(k)$ are the inventories of Manufacturer 1 and Manufacturer 2, respectively; $F_{1}^{t}\left(x_{1}(k)\right)$ and $F_{2}^{s}\left(x_{2}(k)\right)(t, s=$ $1,2)$ are the fuzzy partitions of $x_{1}(k)$ and $x_{2}(k)$, respectively, which meet the conditions of SFP; $D_{0 m}$ is the safety inventory of Manufacturer 1; $D_{1 m}$ is the expected inventory of Manufacturer 1; $D_{0 r}$ is the safety inventory of Manufacturer 2; $D_{1 r}$ is the expected inventory of Manufacturer 2. Let $M_{1}^{1}=M_{1}^{2}=$ $F_{1}^{1}, M_{1}^{3}=M_{1}^{4}=F_{1}^{2}, M_{2}^{1}=M_{2}^{4}=F_{2}^{1}, M_{2}^{2}=M_{2}^{3}=F_{2}^{2}$, $D_{0 m}=8, D_{1 m}=20, D_{0 r}=10$, and $D_{1 r}=25$ ( $\times 10^{3}$ pairs).

As can be seen from Figure 2, this sports shoes supply chain system contains a MORG named $S$ including 4 fuzzy rules $\left(R_{1}, R_{2}, R_{3}\right.$, and $\left.R_{4}\right)$, and 4 fuzzy rules represent the different production strategies and ordering strategies adopted by manufacturers and retailers at different inventory levels. Tables 1 and 2 show the quantitative relations between the compression cost and the compression amount of the lead times for Manufacturer 1 and Manufacturer 2, respectively. Let $c_{m 1}=c_{m 2}=1.78\left(\times 10^{2}\right.$ Yuan $)$. The detailed quantitative relations can be seen in Tables 1 and 2 .

By comparing the stock-out costs data with the data in Tables 1 and 2, we can see that the lead-time compression amounts of Manufacturer 1 and Manufacturer 2 reached the limits of 1.12 days and 0.97 days (i.e., compressing all 4 leadtime components); the stock-out costs $c_{m 1}$ (1.78) and $c_{m 2}(1.78)$ $\left(\times 10^{2}\right.$ Yuan $)$ are greater than the cumulative sums of the compression costs of lead times $c_{\tau 1}(1.415)$ and $c_{\tau 2}(1.222)\left(\times 10^{2}\right.$ Yuan), respectively. Therefore, when the inventory level is less than 0 , according to the cost optimization strategy put forward in Note 1, both Manufacturer 1 and Manufacturer 2 will choose to compress the lead times to minimize the cost of the supply chain. 


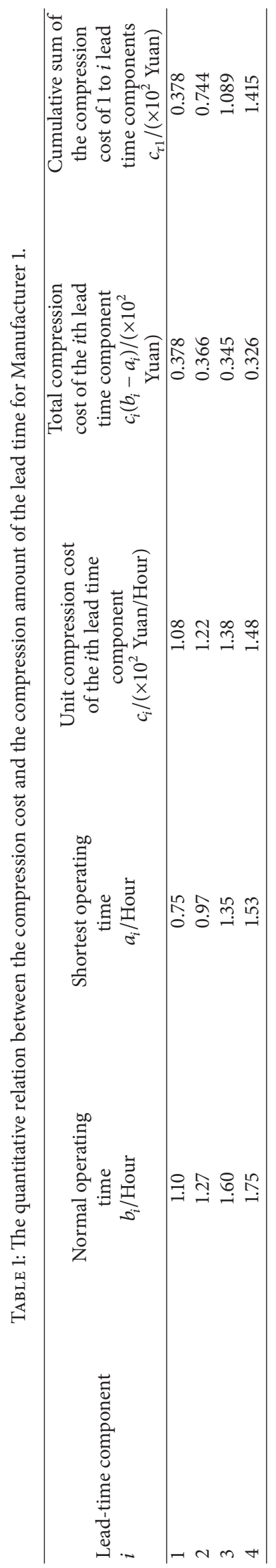




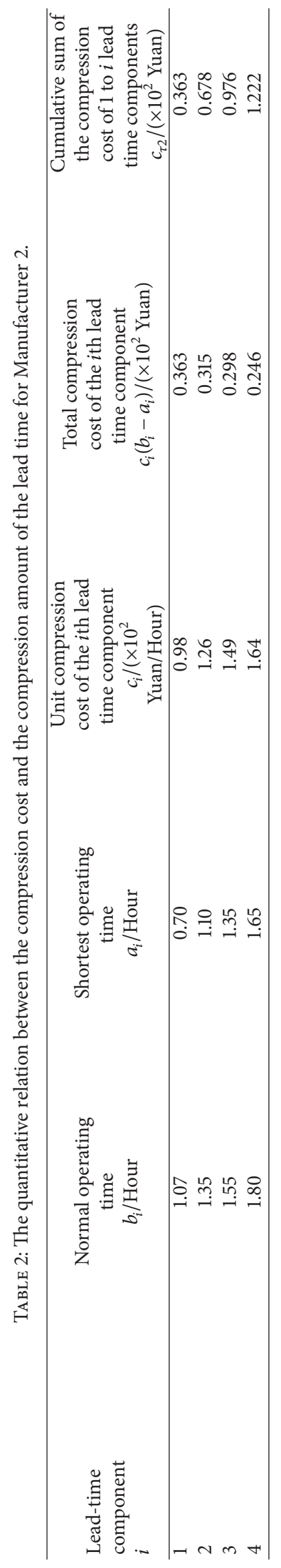


Through the above analysis, the models of the supply chain system with lead times can be expressed as

$$
\begin{aligned}
& R_{1} \\
& x_{1}(k+1)=u_{1}\left(k-\tau_{1}^{\prime \prime}\right)-u_{13}(k), \\
& x_{2}(k+1)=u_{2}\left(k-\tau_{2}^{\prime \prime}\right)-u_{23}(k) \text {, } \\
& x_{3}(k+1)=x_{3}(k)+u_{13}(k)+u_{23}(k)-w_{1}(k), \\
& z(k)=c_{h 3} x_{3}(k)+c_{\tau 1} u_{1}\left(k-\tau_{1}^{\prime \prime}\right) \\
& +c_{\tau 2} u_{2}\left(k-\tau_{2}^{\prime \prime}\right)+c_{s 1} u_{13}(k) \\
& +c_{s 2} u_{23}(k) \text {, } \\
& x_{1}(k+1)=u_{1}\left(k-\tau_{1}^{\prime \prime}\right)-u_{13}(k) \text {, } \\
& x_{2}(k+1)=x_{2}(k)+u_{2}(k)+u_{2}\left(k-\tau_{2}^{\prime}\right)-u_{23}(k) \text {, } \\
& x_{3}(k+1)=x_{3}(k)+u_{13}(k)+u_{23}(k)-w_{1}(k), \\
& z(k)=c_{h 2} x_{2}(k)+c_{h 3} x_{3}(k)+c_{\tau 1} u_{1}\left(k-\tau_{1}^{\prime \prime}\right) \\
& +c_{n 2}\left[u_{2}(k)+u_{2}\left(k-\tau_{2}^{\prime}\right)\right] \\
& +c_{s 1} u_{13}(k)+c_{s 2} u_{23}(k), \\
& x_{1}(k+1)=x_{1}(k)+u_{1}(k)+u_{1}\left(k-\tau_{1}^{\prime}\right)-u_{13}(k), \\
& x_{2}(k+1)=x_{2}(k)+u_{2}(k)+u_{2}\left(k-\tau_{2}^{\prime}\right)-u_{23}(k) \text {, } \\
& x_{3}(k+1)=x_{3}(k)+u_{13}(k)+u_{23}(k)-w_{1}(k), \\
& z(k)=c_{h 1} x_{1}(k)+c_{h 2} x_{2}(k)+c_{h 3} x_{3}(k) \\
& +c_{n 1}\left[u_{1}(k)+u_{1}\left(k-\tau_{1}^{\prime}\right)\right] \\
& +c_{n 2}\left[u_{2}(k)+u_{2}\left(k-\tau_{2}^{\prime}\right)\right] \\
& +c_{s 1} u_{13}(k)+c_{s 2} u_{23}(k) \text {, } \\
& x_{1}(k+1)=x_{1}(k)+u_{1}(k)+u_{1}\left(k-\tau_{1}^{\prime}\right)-u_{13}(k) \text {, } \\
& x_{2}(k+1)=u_{2}\left(k-\tau_{2}^{\prime \prime}\right)-u_{23}(k) \text {, } \\
& x_{3}(k+1)=x_{3}(k)+u_{13}(k)+u_{23}(k)-w_{1}(k), \\
& z(k)=c_{h 1} x_{1}(k)+c_{h 3} x_{3}(k) \\
& +c_{n 1}\left[u_{1}(k)+u_{1}\left(k-\tau_{1}^{\prime}\right)\right] \\
& +c_{\tau 2} u_{2}\left(k-\tau_{2}^{\prime \prime}\right)+c_{s 1} u_{13}(k) \\
& +c_{s 2} u_{23}(k) \text {. }
\end{aligned}
$$

The above 4 different fuzzy rules $\left(R_{1}, R_{2}, R_{3}\right.$, and $\left.R_{4}\right)$ represent the different strategies taken by manufacturers at different inventory levels. The details of the rules can be expressed as follows: $R_{1}$ : both Manufacturer 1 and Manufacturer 2 take the lead-time compression strategy, that is, the emergency production of sports shoes; $R_{2}$ : Manufacturer 1 takes the lead-time compression strategy and Manufacturer 2 produces the sports shoes normally; $R_{3}$ : both Manufacturer 1 and Manufacturer 2 produce the sports shoes normally; $R_{4}$ : Manufacturer 1 produces the sports shoes normally and Manufacturer 2 takes the lead-time compression strategy. In the above 4 rules, the retailers can order the sports shoes normally.

The above supply chain models under the different rules can be converted into the following Takagi-Sugeno fuzzy model:

$R^{i}:$ if $x_{1}(k)$ is $M_{1}^{i}$ and $x_{2}(k)$ is $M_{2}^{i}$, then

$$
\begin{aligned}
& \mathbf{x}(k+1)=\sum_{i=1}^{4} h_{i}\left[\mathbf{A}_{i} \mathbf{x}(k)+\mathbf{B}_{i} \mathbf{u}(k)\right. \\
& \left.+\sum_{a=1}^{2 \times(3-1)} \mathbf{B}_{i a} \mathbf{u}\left(k-\tau_{a}\right)+\mathbf{B}_{w i} \mathbf{w}(k)\right], \\
& z(k)=\sum_{i=1}^{4} h_{i}\left[\mathbf{C}_{i} \mathbf{x}(k)+\mathbf{D}_{i} \mathbf{u}(k)\right. \\
& \left.+\sum_{a=1}^{2 \times(3-1)} \mathbf{D}_{i a} \mathbf{u}\left(k-\tau_{a}\right)\right]
\end{aligned}
$$

Based on the above Takagi-Sugeno fuzzy model, the fuzzy state feedback controller of the system can be designed as follows:

$K^{i}:$ if $x_{1}(k)$ is $M_{1}^{i}$ and $x_{2}(k)$ is $M_{2}^{i}$, then

$$
\begin{gathered}
\mathbf{u}(k)=-\sum_{i=1}^{4} h_{i} \mathbf{K}_{i 1} \mathbf{x}(k), \\
\mathbf{u}_{1}\left(k-\tau_{1}^{\prime}\right)=-\sum_{i=1}^{4} h_{i} \mathbf{K}_{i 11} \mathbf{x}\left(k-\tau_{1}^{\prime}\right), \\
\mathbf{u}_{2}\left(k-\tau_{2}^{\prime}\right)=-\sum_{i=1}^{4} h_{i} \mathbf{K}_{i 21} \mathbf{x}\left(k-\tau_{2}^{\prime}\right), \\
\mathbf{u}_{1}\left(k-\tau_{1}^{\prime \prime}\right)=-\sum_{i=1}^{4} h_{i} \mathbf{K}_{i 31} \mathbf{x}\left(k-\tau_{1}^{\prime \prime}\right), \\
\mathbf{u}_{2}\left(k-\tau_{2}^{\prime \prime}\right)=-\sum_{i=1}^{4} h_{i} \mathbf{K}_{i 41} \mathbf{x}\left(k-\tau_{2}^{\prime \prime}\right) .
\end{gathered}
$$

Based on the actual operating data, all parameters are set as follows:

$$
\begin{aligned}
& c_{h 1}=0.015\left(\times 10^{2} \text { Yuan }\right), \\
& c_{h 2}=0.018\left(\times 10^{2} \text { Yuan }\right), \\
& c_{h 3}=0.020\left(\times 10^{2} \text { Yuan }\right), \\
& c_{n 1}=1.10\left(\times 10^{2} \text { Yuan }\right),
\end{aligned}
$$




$$
\begin{aligned}
& c_{n 2}=1.20\left(\times 10^{2} \text { Yuan }\right) \text {, } \\
& c_{s 1}=1.25\left(\times 10^{2} \text { Yuan }\right) \text {, } \\
& c_{s 2}=1.40\left(\times 10^{2} \text { Yuan }\right) \text {, } \\
& c_{m 1}=c_{m 2}=1.78\left(\times 10^{2} \text { Yuan }\right) \text {, } \\
& c_{\tau 1}=1.415\left(\times 10^{2} \text { Yuan }\right) \text {, } \\
& c_{\tau 2}=1.222\left(\times 10^{2} \text { Yuan }\right) \text {, } \\
& \mathbf{A}_{1}=\left[\begin{array}{lll}
0 & 0 & 0 \\
0 & 0 & 0 \\
0 & 0 & 1
\end{array}\right], \\
& \mathbf{A}_{2}=\left[\begin{array}{lll}
0 & 0 & 0 \\
0 & 1 & 0 \\
0 & 0 & 1
\end{array}\right] \text {, } \\
& \mathbf{A}_{3}=\left[\begin{array}{lll}
1 & 0 & 0 \\
0 & 1 & 0 \\
0 & 0 & 1
\end{array}\right] \text {, } \\
& \mathbf{B}_{22}=\mathbf{B}_{32}=\left[\begin{array}{llll}
0 & 0 & 0 & 0 \\
0 & 1 & 0 & 0 \\
0 & 0 & 0 & 0
\end{array}\right] \text {, } \\
& \mathbf{B}_{13}=\mathbf{B}_{23}=\left[\begin{array}{llll}
0 & 0 & 1 & 0 \\
0 & 0 & 0 & 0 \\
0 & 0 & 0 & 0
\end{array}\right] \text {, } \\
& \mathbf{B}_{33}=\mathbf{B}_{43}=\left[\begin{array}{llll}
0 & 0 & 0 & 0 \\
0 & 0 & 0 & 0 \\
0 & 0 & 0 & 0
\end{array}\right] \text {, } \\
& \mathbf{B}_{14}=\mathbf{B}_{44}=\left[\begin{array}{llll}
0 & 0 & 0 & 0 \\
0 & 0 & 0 & 1 \\
0 & 0 & 0 & 0
\end{array}\right] \text {, } \\
& \mathbf{B}_{24}=\mathbf{B}_{34}=\left[\begin{array}{llll}
0 & 0 & 0 & 0 \\
0 & 0 & 0 & 0 \\
0 & 0 & 0 & 0
\end{array}\right] \text {, } \\
& \mathbf{A}_{4}=\left[\begin{array}{lll}
1 & 0 & 0 \\
0 & 0 & 0 \\
0 & 0 & 1
\end{array}\right] \text {, } \\
& \mathbf{B}_{1}=\left[\begin{array}{cccc}
0 & 0 & -1 & 0 \\
0 & 0 & 0 & -1 \\
0 & 0 & 1 & 1
\end{array}\right] \text {, } \\
& \mathbf{B}_{2}=\left[\begin{array}{cccc}
0 & 0 & -1 & 0 \\
0 & 1 & 0 & -1 \\
0 & 0 & 1 & 1
\end{array}\right] \text {, } \\
& \mathbf{B}_{3}=\left[\begin{array}{cccc}
1 & 0 & -1 & 0 \\
0 & 1 & 0 & -1 \\
0 & 0 & 1 & 1
\end{array}\right] \text {, } \\
& \mathbf{B}_{4}=\left[\begin{array}{cccc}
1 & 0 & -1 & 0 \\
0 & 0 & 0 & -1 \\
0 & 0 & 1 & 1
\end{array}\right] \text {, } \\
& \mathbf{B}_{11}=\mathbf{B}_{21}=\left[\begin{array}{llll}
0 & 0 & 0 & 0 \\
0 & 0 & 0 & 0 \\
0 & 0 & 0 & 0
\end{array}\right] \text {, } \\
& \mathbf{B}_{31}=\mathbf{B}_{41}=\left[\begin{array}{llll}
1 & 0 & 0 & 0 \\
0 & 0 & 0 & 0 \\
0 & 0 & 0 & 0
\end{array}\right] \text {, } \\
& \mathbf{B}_{12}=\mathbf{B}_{42}=\left[\begin{array}{llll}
0 & 0 & 0 & 0 \\
0 & 0 & 0 & 0 \\
0 & 0 & 0 & 0
\end{array}\right] \text {, } \\
& \mathbf{B}_{w 1}=\mathbf{B}_{w 2}=\mathbf{B}_{w 3}=\mathbf{B}_{w 4}=\left[\begin{array}{ccc}
0 & 0 & 0 \\
0 & 0 & 0 \\
0 & 0 & -1
\end{array}\right] \text {, } \\
& \mathbf{C}_{1}=\left[\begin{array}{lll}
0 & 0 & c_{h 3}
\end{array}\right], \\
& \mathbf{C}_{2}=\left[\begin{array}{lll}
0 & c_{h 2} & c_{h 3}
\end{array}\right], \\
& \mathbf{C}_{3}=\left[\begin{array}{lll}
c_{h 1} & c_{h 2} & c_{h 3}
\end{array}\right], \\
& \mathbf{C}_{4}=\left[\begin{array}{lll}
c_{h 1} & 0 & c_{h 3}
\end{array}\right] \text {, } \\
& \mathbf{D}_{1}=\left[\begin{array}{llll}
0 & 0 & c_{s 1} & c_{s 2}
\end{array}\right] \text {, } \\
& \mathbf{D}_{2}=\left[\begin{array}{llll}
0 & c_{n 2} & c_{s 1} & c_{s 2}
\end{array}\right] \text {, } \\
& \mathbf{D}_{3}=\left[\begin{array}{llll}
c_{n 1} & c_{n 2} & c_{s 1} & c_{s 2}
\end{array}\right], \\
& \mathbf{D}_{4}=\left[\begin{array}{llll}
c_{n 1} & 0 & c_{s 1} & c_{s 2}
\end{array}\right] \text {, } \\
& \mathbf{D}_{11}=\mathbf{D}_{21}=\left[\begin{array}{lll}
0 & 0 & 0
\end{array}\right] \text {, } \\
& \mathbf{D}_{31}=\mathbf{D}_{41}=\left[\begin{array}{llll}
c_{n 1} & 0 & 0 & 0
\end{array}\right] \text {, } \\
& \mathbf{D}_{12}=\mathbf{D}_{42}=\left[\begin{array}{llll}
0 & 0 & 0 & 0
\end{array}\right] \text {, } \\
& \mathbf{D}_{22}=\mathbf{D}_{32}=\left[\begin{array}{llll}
0 & c_{n 2} & 0 & 0
\end{array}\right] \text {, } \\
& \mathbf{D}_{13}=\mathbf{D}_{23}=\left[\begin{array}{llll}
0 & 0 & c_{\tau 1} & 0
\end{array}\right] \text {, } \\
& \mathbf{D}_{33}=\mathbf{D}_{43}=\left[\begin{array}{llll}
0 & 0 & 0 & 0
\end{array}\right] \text {, } \\
& \mathbf{D}_{14}=\mathbf{D}_{44}=\left[\begin{array}{llll}
0 & 0 & 0 & c_{\tau 2}
\end{array}\right] \text {, } \\
& \mathbf{D}_{24}=\mathbf{D}_{34}=\left[\begin{array}{llll}
0 & 0 & 0 & 0
\end{array}\right] \text {, } \\
& \gamma=0.95 \text {. }
\end{aligned}
$$




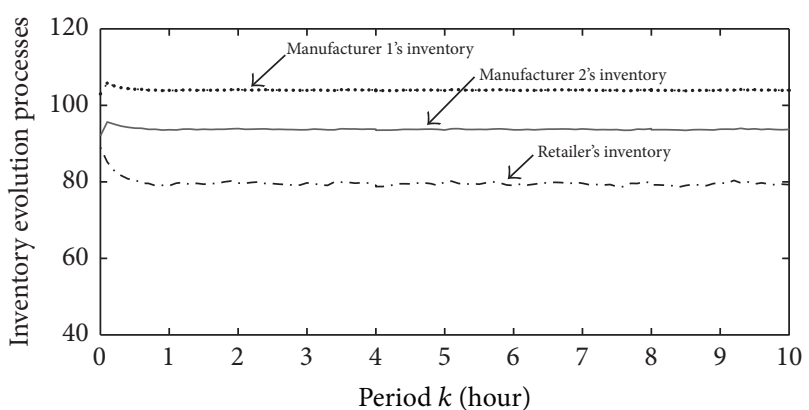

FIGURE 3: Evolution processes of inventories with cost optimization strategy $\left(\times 10^{3}\right.$ pairs $)$.

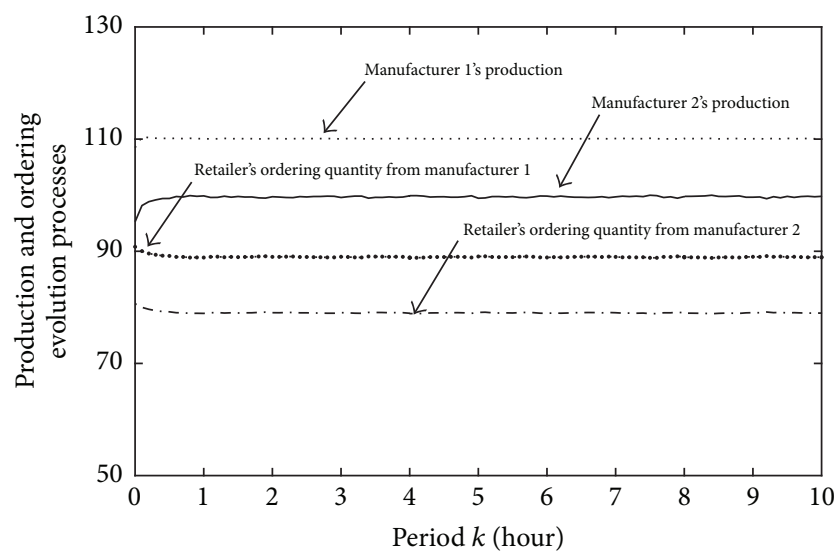

FIGURE 4: Evolution processes of productions and ordering quantities with cost optimization strategy $\left(\times 10^{3}\right.$ pairs $)$.

By solving the inequalities (10) in Theorem 3, we can get the following results:

$$
\begin{aligned}
\mathbf{P}_{1} & =\left[\begin{array}{ccc}
62.2597 & 0.0149 & 0.0663 \\
0.0149 & 62.2581 & 0.0637 \\
0.0663 & 0.0637 & 62.5223
\end{array}\right], \\
\mathbf{Q}_{11} & =\mathbf{Q}_{21}=\mathbf{Q}_{31}=\mathbf{Q}_{41} \\
& =\left[\begin{array}{ccc}
12.4471 & -0.0001 & -0.0003 \\
-0.0001 & 12.4471 & -0.0003 \\
-0.0003 & -0.0003 & 12.4460
\end{array}\right] .
\end{aligned}
$$

The above results satisfy the robust stability conditions of Theorem 3, so we know that the supply chain system with lead times is robustly stable. In addition, the supply chain system is described by the actual values; that is, the simulation results are equal to the deviation values plus the normal values. Let the initial values be $x_{1}(0)=-2, x_{2}(0)=-3$, and $x_{3}(0)=4$ $\left(\times 10^{3}\right.$ pairs); the normal values be $\vec{x}_{1}(k)=105, \vec{x}_{2}(k)=$ $95, \vec{x}_{3}(k)=85, \vec{u}_{1}(k)=110, \vec{u}_{2}(k)=98, \vec{u}_{13}(k)=$ 90 , and $\vec{u}_{23}(k)=80\left(\times 10^{3}\right.$ pairs $)$. Suppose the customers' demands follow the normal distribution disturbance, that is, $w_{1}(k) \sim N\left(6,0.8^{2}\right)$. The simulation results are shown in Figures 3-6, where Figures 3-5 show the simulation results of

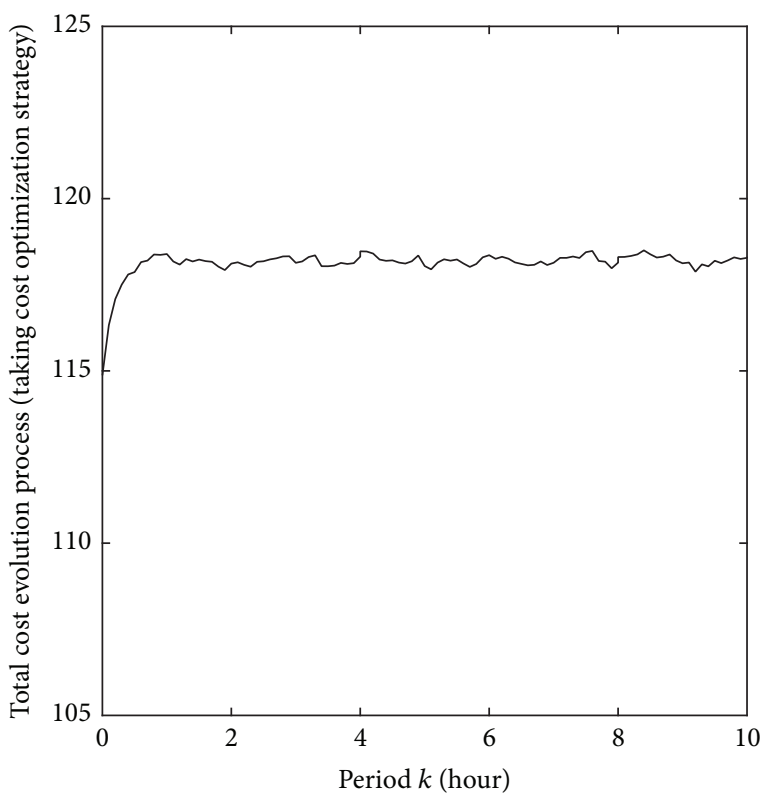

FIGURE 5: Evolution process of total cost with cost optimization strategy $\left(\times 10^{5}\right.$ Yuan $)$.

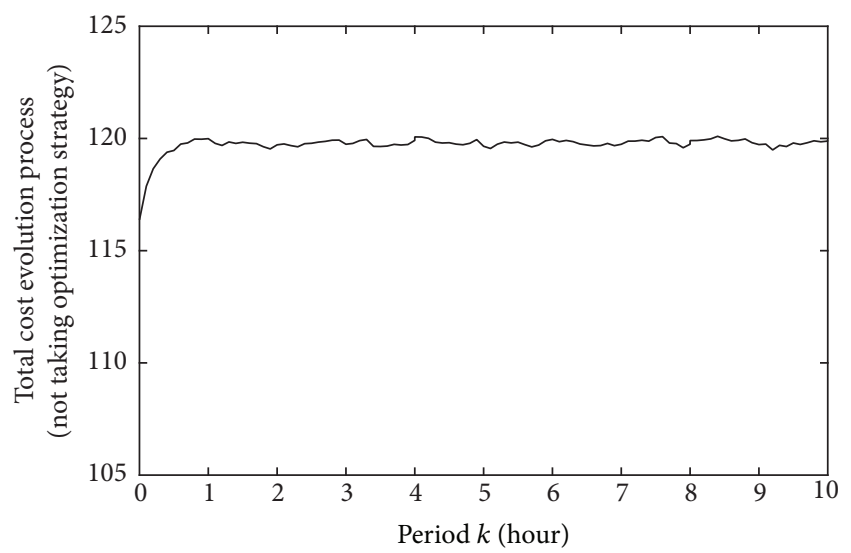

FIGURE 6: Evolution process of total cost without cost optimization strategy $\left(\times 10^{5}\right.$ Yuan $)$.

the inventory levels, the productions, the ordering quantities, and the total cost with the cost optimization strategy, and Figure 6 shows the simulation result of the total cost without the cost optimization strategy.

It can be seen from Figures 3-6 that the fuzzy robust control strategy proposed in this paper makes the state variables, control variables, and total cost fluctuate in a small range and ensures the stable operation of the whole system. According to Note 1, the total cost of the supply chain with the cost optimization strategy can be expressed as $z(k)=$ $\sum_{a=1}^{n-1}\left\{c_{h a} x_{a}(k)+c_{\tau a} u_{a}\left(k-\tau_{a}^{\prime \prime}\right)+c_{s a} u_{a n}(k)\right\}+c_{h n} x_{n}(k)$, while the total cost of the supply chain without the cost optimization strategy can be expressed as $z(k)=\sum_{a=1}^{n-1}\left\{c_{h a} x_{a}(k)+c_{n a}\left[u_{a}(k)+\right.\right.$ $\left.\left.u_{a}\left(k-\tau_{a}^{\prime}\right)\right]+c_{m a} u_{a n}(k)\right\}+c_{h n} x_{n}(k)$. Therefore, it can be seen from the comparison between Figures 5 and 6 that the cost 
optimization strategy adopted in different inventory levels can effectively reduce the total cost of the supply chain system.

\section{Conclusion}

In this paper, we have constructed a dynamic supply chain model with lead times in consideration of the inventory levels, production strategy, ordering strategy, lead-time compression cost, stock-out cost, and customers' demands in supply chain node enterprises and proposed the cost optimization strategy and the fuzzy robust control strategy for the dynamic model. Through the comparison and analysis of the simulation results, we get the following conclusions: (1) compared to the supply chain without the cost optimization strategy, the supply chain with the cost optimization strategy can reduce the total cost of the supply chain system effectively; (2) the proposed fuzzy robust control strategy can effectively suppress the impacts of the external stochastic demands and lead time disturbances on the supply chain system and guarantee the stable operation of the supply chain system.

\section{Conflicts of Interest}

The authors declare that they have no conflicts of interest.

\section{Acknowledgments}

This work was supported by the National Social Science Foundation of China (17BGL222).

\section{References}

[1] H. Huang, H. Ke, and L. Wang, "Equilibrium analysis of pricing competition and cooperation in supply chain with one common manufacturer and duopoly retailers," International Journal of Production Economics, vol. 178, pp. 12-21, 2016.

[2] M. H. Lotfi and S. Sarkar, "Uncertain price competition in a duopoly with heterogeneous availability," Institute of Electrical and Electronics Engineers Transactions on Automatic Control, vol. 61, no. 4, pp. 1010-1025, 2016.

[3] D. Yang, E. Qi, and Y. Li, "Quick response and supply chain structure with strategic consumers," Omega, vol. 52, pp. 1-14, 2015.

[4] R. Dou, C. Zong, and M. Li, "An interactive genetic algorithm with the interval arithmetic based on hesitation and its application to achieve customer collaborative product configuration design," Applied Soft Computing, vol. 38, pp. 384-394, 2016.

[5] Q. Bai, M. Chen, and L. Xu, "Revenue and promotional costsharing contract versus two-part tariff contract in coordinating sustainable supply chain systems with deteriorating items," International Journal of Production Economics, vol. 187, pp. 85101, 2017.

[6] E. Kim and T. Park, "Admission and inventory control of a single-component make-to-order production system with replenishment setup cost and lead time," European Journal of Operational Research, vol. 255, no. 1, pp. 91-102, 2016.

[7] H.-J. Lin, "Investing in lead-time variability reduction in a collaborative vendor-buyer supply chain model with stochastic lead time," Computers \& Operations Research, vol. 72, pp. 43-49, 2016.

[8] A. Arkan and S. R. Hejazi, "Coordinating orders in a two echelon supply chain with controllable lead time and ordering cost using the credit period," Computers \& Industrial Engineering, vol. 62, no. 1, pp. 56-69, 2012.

[9] C. H. Glock, "Lead time reduction strategies in a singlevendorsingle-buyer integrated inventory model with lot sizedependent lead times and stochastic demand," International Journal of Production Economics, vol. 136, no. 1, pp. 37-44, 2012.

[10] S. Cannella, R. Dominguez, and J. M. Framinan, "Inventory record inaccuracy - The impact of structural complexity and lead time variability," OMEGA - The International Journal of Management Science, vol. 68, pp. 123-138, 2017.

[11] J. C. Hayya, T. P. Harrison, and X. J. He, "The impact of stochastic lead time reduction on inventory cost under order crossover," European Journal of Operational Research, vol. 211, no. 2, pp. 274-281, 2011.

[12] L. Teng, Y. Wang, W. Cai, and H. Li, "Robust model predictive control of discrete nonlinear systems with time delays and disturbances via T-S fuzzy approach," Journal of Process Control, vol. 53, pp. 70-79, 2017.

[13] A. Aalaei and H. Davoudpour, "A robust optimization model for cellular manufacturing system into supply chain management," International Journal of Production Economics, vol. 183, pp. 667679, 2017.

[14] X. Xiang, C. Yu, and Q. Zhang, "Robust fuzzy 3D path following for autonomous underwater vehicle subject to uncertainties," Computers \& Operations Research, vol. 84, pp. 165-177, 2017.

[15] O. Solyali, J. F. Cordeau, and G. Laporte, "The impact of modeling on robust inventory management under demand uncertainty," Management Science, vol. 62, no. 4, pp. 1188-1201, 2016.

[16] S. Zhang, X. Li, and C. Y. Zhang, "A fuzzy control model for restraint of bullwhip effect in uncertain closed-loop supply chain with hybrid recycling channels," IEEE Transactions on Fuzzy Systems, vol. 25, no. 2, pp. 475-482, 2017.

[17] X. B. Xiang, C. Y. Yu, L. Lapierre, J. L. Zhang, and Q. Zhang, "Survey on fuzzy-logic-based guidance and control of marine surface vehicles and underwater vehicles," International Journal of Fuzzy Systems, pp. 1-15, 2017.

[18] G. Feng, "A survey on analysis and design of model-based fuzzy control systems," IEEE Transactions on Fuzzy Systems, vol. 14, no. 5, pp. 676-697, 2006.

[19] Y. D. He, Z. He, D. H. Lee, K. J. Kim, L. Zhang, and X. X. Yang, "Robust fuzzy programming method for MRO problems considering location effect, dispersion effect and model uncertainty," Computers Industrial Engineering, vol. 105, pp. 76-83, 2017.

[20] Z.-H. Xiu and G. Ren, "Stability analysis and systematic design of Takagi-Sugeno fuzzy control systems," Fuzzy Sets and Systems, vol. 151, no. 1, pp. 119-138, 2005.

[21] S. T. Zhang, Y. T. Hou, S. Q. Zhang, and M. Zhang, "Fuzzy control model and simulation for nonlinear supply chain system with lead times," Complexity, vol. 2017, 11 pages, 2017. 


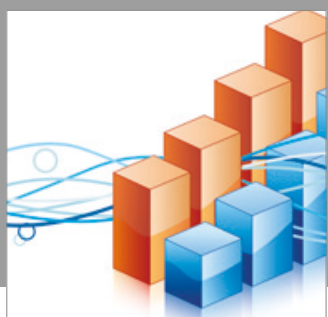

Advances in

Operations Research

vatersals

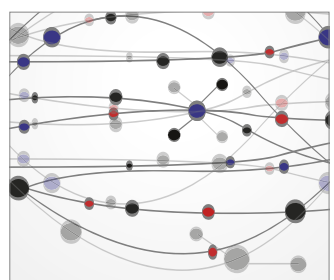

\section{The Scientific} World Journal
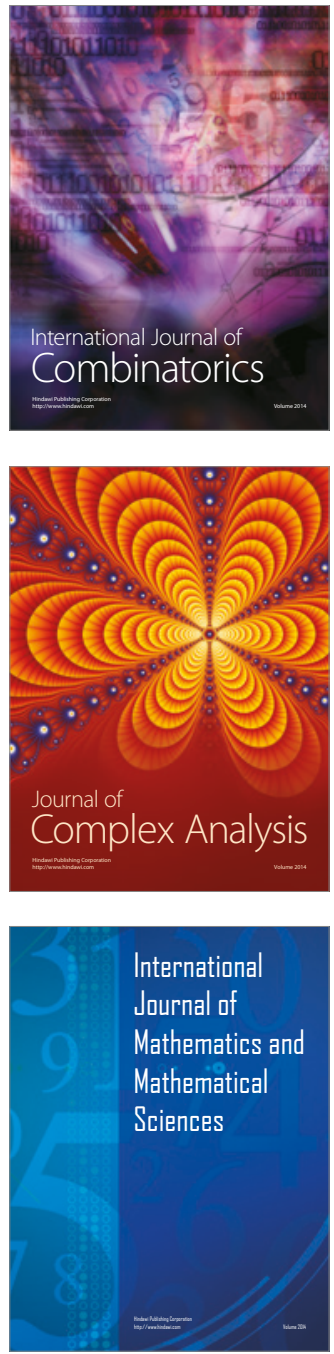
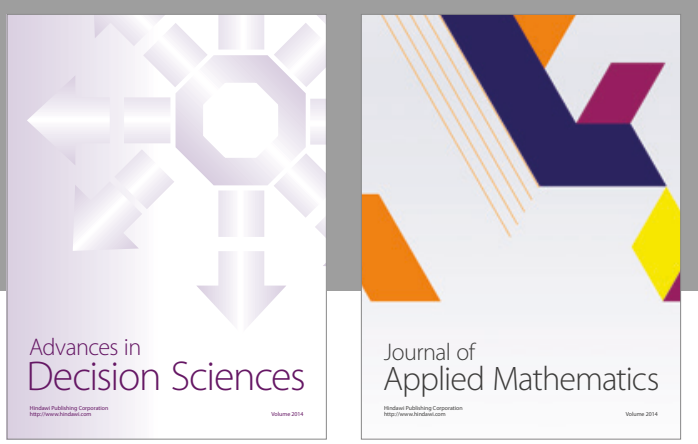

Algebra

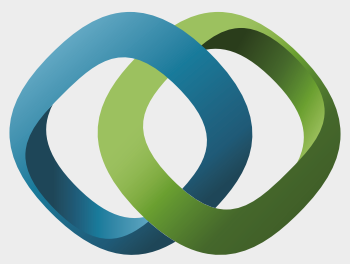

\section{Hindawi}

Submit your manuscripts at

https://www.hindawi.com
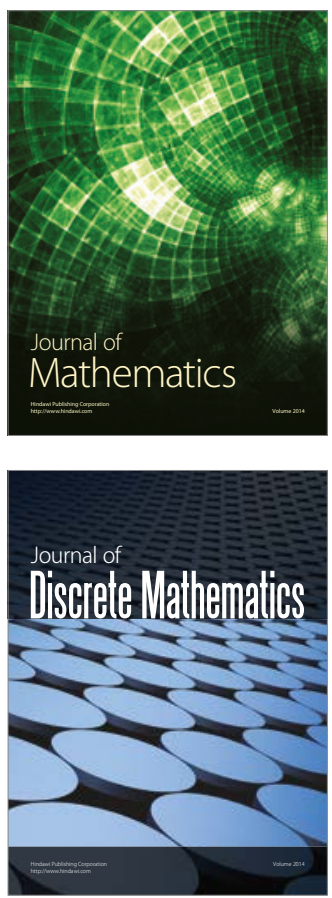

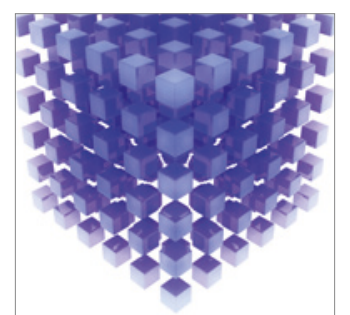

Mathematical Problems in Engineering
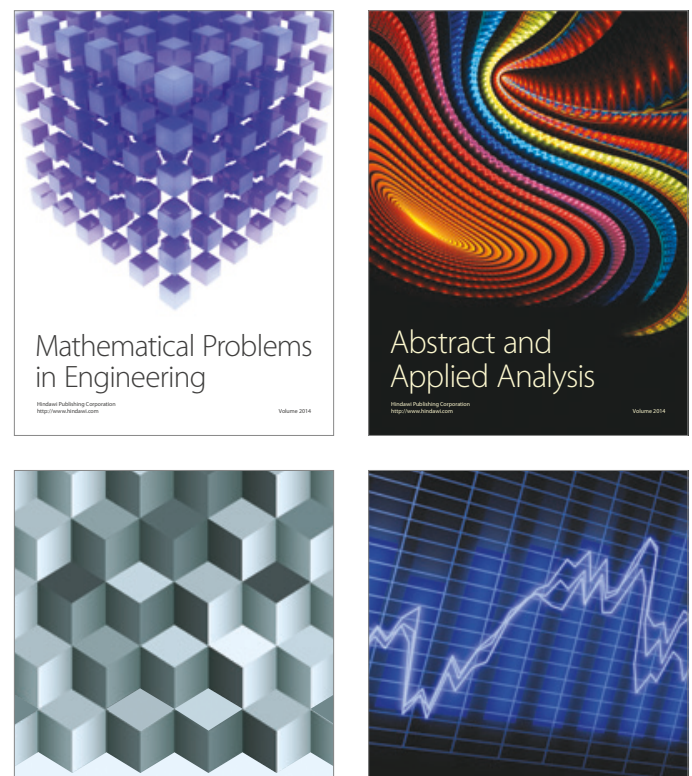

Journal of

Function Spaces

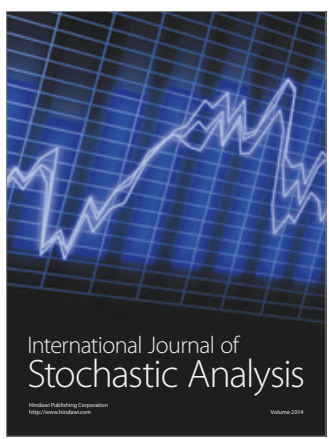

Probability and Statistics
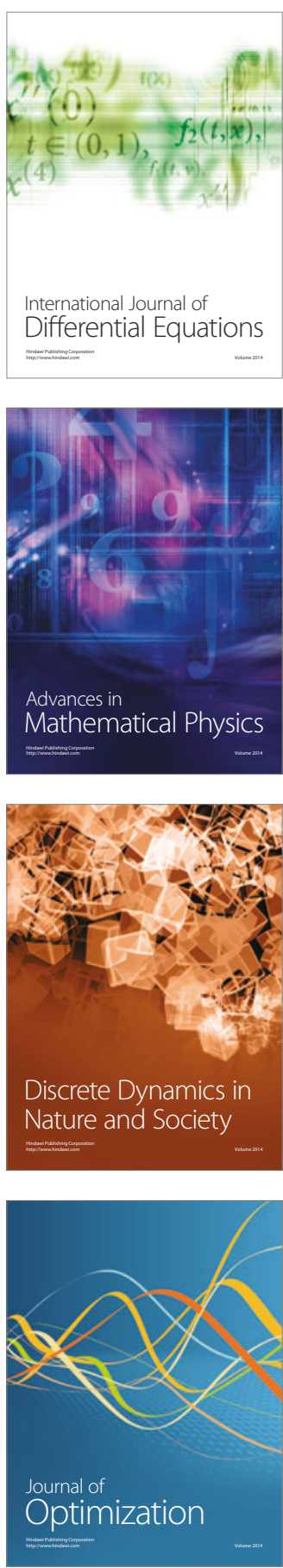